\title{
An unusual de novo duplication 10p/deletion 10q syndrome: The first case in Korea
}

Bom-Yi Lee, Ju-Yeon Park', Yeon-Woo Lee', Ah-Rum Oh', Shin-Young Lee', Eun-Young Choi', Moon-Young Kim², Hyun-Mee Ryu ${ }^{1,2}$, and So-Yeon Park ${ }^{1, *}$

'Laboratory of Medical Genetics, Medical Research Institute, ${ }^{2}$ Department of Obstetrics and Gynecology, Cheil General Hospital \& Women's Healthcare Center, Seoul, Korea

We herein report an analysis of a female baby with a de novo dup(10p)/del(10q) chromosomal aberration. A prenatal cytogenetic analysis was performed owing to abnormal ultrasound findings including a choroid plexus cyst, prominent cisterna magna, and a slightly medially displaced stomach. The fetal karyotype showed additional material attached to the terminal region of chromosome 10q. Parental karyotypes were both normal. At birth, the baby showed hypotonia, upslanting palpebral fissures, a nodular back mass, respiratory distress, neonatal jaundice and a suspicious polycystic kidney. We ascertained that the karyotype of the baby was $46, X X, \operatorname{der}(10)($ pter $\rightarrow q 26.3:: p 11.2 \rightarrow$ pter) by cytogenetic and molecular cytogenetic analyses including high resolution GTG-and RBG-banding, fluorescence in situ hybridization, comparative genomic hybridization, and short tandem repeat marker analyses. While almost all reported cases of 10p duplication originated from one of the parents with a pericentric inversion, our case is extraordinarily rare as the de novo dup(10p)/ del(10q) presumably originated from a rearrangement at the premeiotic stage of the parental germ cell or from parental germline mosaicism.

Key words: Chromosome 10, trisomy 10p, Chromosome 10, monosomy 10q.

\section{Introduction}

Dup(10p)/del(10q) aberrations are usually generated from recombination between a parental pericentric inverted chromatid and its normal homologue during meiosis I. Cases of dup(10p) with small del(10q), with respect to clinical phenotypes, are regarded as trisomy 10p syndrome because they involve a large segment of chromosome 10p [1-3].

Most previous trisomy 10p cases originated from familial reciprocal translocations or familial pericentric inversions and de novo trisomy 10 is very rare [4-6]. The common clinical features of trisomy $10 p$ are severe mental and growth retardation, craniofacial anomalies (high forehead, hypertelorism, upward slant of the palpebral fissures, and cleft lip and palate), and other organ malformations (cardiac and renal anomaly) and skeletal abnormalities (clubfoot and flexion abnormalities).

Since Lewandowsky et al. [7] reported the deletion in the long arm of chromosome 10, over 60 cases of interstitial or terminal $10 q$ deletion ranging from $10 q 23-q 26$ resulting from familial translocations or other chromosomal anomalies have been identified. The main clinical manifestations of terminal deletion of $10 q$ syndrome are typical facial dysmorphisms, mental and

Received: 27 April 2015, Revised: 19 May 2015, Accepted: 8 June 2015, Published: 30 June 2015

*Corresponding author: So-Yeon Park, Ph.D.

Laboratory of Medical Genetics, Medical Research Institute, Cheil General Hospital \&t Women's Healthcare Center, 17 Seoae-ro 1-gil, Jung-gu, Seoul 100-380, Korea.

Tel: +82-2-2000-7680, Fax: +82-2-2278-4574, E-mail: paranip5@hanmail.net

Conflict of interest: We declare that we do not have any conflicts of interests.

(c) This is an open-access article distributed under the terms of the Creative Commons Attribution Non-Commercial License (http://creativecommons.org/licenses/by-nc/4.0/) which permits unrestricted non-commercial use, distribution, and reproduction in any medium, provided the original work is properly cited.

(c) Copyright 2015 by the Korean Society of Medical Genetics

www.e-kjgm.org 
developmental retardation, postnatal growth retardation, anogenital anomalies, limb abnormalities or cardiac/renal anomalies.

Fourteen cases of rec(10), duplication 10p accompanied with deletion 10q, have been reported previously, and all were derived from a parental pericentric inversion.

Here, we report an exceptional de novo dup(10p)/del(10q) case characterized by high resolution (HR) GTG- and RBGbanding, fluorescence in situ hybridization (FISH), comparative genomic hybridization (CGH) techniques, and short tandem repeat (STR) marker analyses; a few common phenotypic features were observed based on a review of previous cases of rec(10). This study was approved by the Ethics Committee of Cheil General Hospital and Women's Health Care Center (\#CGHIRBGR-2007-02) and patient's consent was obtained.

\section{Case}

\section{Case description}

A 24-year-old, mother (G1 P0) was referred for cordocentesis owing to abnormal ultrasound findings including right choroid plexus cysts, prominent cisterna magna, slightly medially displaced stomach, and renal pelvis dilation at 26 weeks of gestation. After genetic counseling, parental chromosome analyses were also carried out. A female baby was born at 38.2 weeks of gestation by normal vaginal delivery. The birth weight was 2,910 g (50th percentile), length was $47 \mathrm{~cm}$ (25th percentile), and head circumference was $33 \mathrm{~cm}$ (25-50th percentile). The Apgar scores were 6 and 7 at 1 and 5 min, respectively. Four days after delivery, she was transferred to the newborn intensive care unit owing to hypotonia with decreased muscle tone and moderate feeding difficulties. General activity was good and neck and clavicles were normal. The urogenital tract and anus were normal, and extremity and motor activity was good. However, the newborn baby had a 1-cm nodular back mass, up-slanting palpebral fissures, asymmetric head shape, and suspicious multiple renal cortical cysts in both kidneys. In addition, the baby exhibited respiratory distress, neonatal jaundice, unspecified thrombocytopenia, and direct bilirubinemia. On day 25 , the baby was discharged from the hospital.

\section{Cytogenetic and $\mathrm{CGH}$ analysis}

Conventional GTG-banding analyses were carried out on cultured lymphocytes obtained from fetal cord blood and parental peripheral blood samples. After birth, HR GTG- and RBG-banding techniques were performed to determine the precise breakpoints of the abnormal chromosome.

The genomic DNAs for CGH analyses were extracted from peripheral blood samples of the infant and from a karyotypically normal female for use as a control (reference DNA). CGH procedures were performed as described by Kallioniemi et al. [8] and the manufacturer's instructions (Vysis Inc., Downers Grove, IL, USA). CGH signals were captured (ZEISS, Oberkochen, Germany) and analyzed by the CytoVision (version 3.52; Applied Imaging, Southend-on-Sea, UK).

All 30 metaphase chromosomes from the fetal cord blood sample indicated that the fetus had extra chromosomal material at the long arm of one chromosome 10. Initially, we reported the fetal karyotype as 46,XX,add(10)(q?25). The results of the parental chromosome study were both normal indicating the de novo origin of the abnormal fetal chromosome 10. After birth, we reexamined the newborn's chromosome obtained from the lymphocyte culture using HR GTG- and RBG-banding
A Normal 10
Dup 10p
B
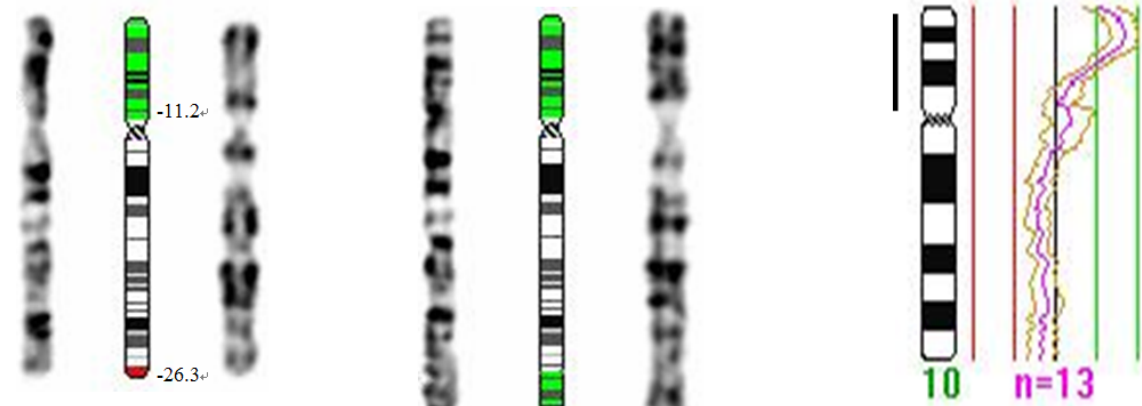
Fig. 1. Partial karyotype and ideogram and comparative genomic hybridization (CGH) analysis. (A) GTG- (left) and RBG- (right) banded partial karyotype and ideogram (middle) of the duplicated chromosome 10 of the infant showing the short arm of chromosome 10 attached to the terminal region of the long arm of chromosome 10. 46,XX,der(10)(pter $\rightarrow$ q26.3::p11.2 $\rightarrow$ pter). (B) CGH analysis. Partial profile of dup(10p) shows amplification of the signal at the chromosome 10 pter $\rightarrow$ p11.2 segment (black bar). 
techniques, and revealed that the chromosome 10p was duplicated (Fig. 1A).

The $\mathrm{CGH}$ profile showed prominent amplification of the signal in the region of $10 p 11.2 \rightarrow 15.3$. However, signal loss in the region of 10q26.3 was not detected (Fig. 1B). The deleted region was likely too small to detect by $\mathrm{CGH}$. After collecting the above data, we defined the newborn's karyotype as 46,XX,der(10) (pter $\rightarrow$ q26.3::p11.2 $\rightarrow$ pter).

\section{FISH analysis}

FISH studies on the metaphase chromosomes obtained from the newborn's lymphocyte culture were carried out to identify both ends of chromosome 10. Two probes for the subtelomeric region of 10p (TelVysion 10p Spectrum Green; Vysis Inc.) and 10q (TEL 10q DNA Probe Red; Qbiogene, Amsterdam, The Netherlands) were used according to the manufacturer's instructions. The FISH images were observed under an Axio Imager Z1 (ZEISS) and captured using Isis software (ver. 5.1.2; MetaSystems, Altlussheim, Germany).

FISH studies with the probe for subtelomeric regions of $10 p$ and $10 q$ showed two signals for 10p in both ends of the abnormal chromosome 10 (duplication of 10p) and loss of signal for $10 q$ (deletion of 10q) (Fig. 2).

\section{STR marker analysis}

STR marker analyses were performed to examine the parental origin of the duplicated region on the infant's chromosome $10 p$ by using 10 STR marker loci. The highly heterozygotic STR markers D10S526, D10S1145, D10S518, D10S504, D10S1154, D10S1142, D10S514, D10S1168, D10S527, and D10S2325 were selected residing from 10p15 through 10p12, from the Ensemble Genome Browser (http://www.ensemble.org) and the National Center for Biotechnology Information (NCBI) genome database (http://www.ncbi.nlm.nih.gov). A 20 ng sample of DNA from each family member was amplified in a $15 \mu \mathrm{L}$ reaction containing the following: $10 \mathrm{mM}$ Tris- $\mathrm{HCl}, 50 \mathrm{mM} \mathrm{KCl}, 1.5 \mathrm{mM}$ $\mathrm{MgCl}_{2}, 10 \mathrm{mM}$ dNTP (ABI, Walthanm, MA, USA), 0.2 U GoldTaq polymerase (Perkin Elmer, Walthanm, MA, USA), and $0.67 \mathrm{pmol}$ each primer. Amplification was performed with the following conditions: 5 min at $95^{\circ} \mathrm{C}, 35$ cycles of 30 s of denaturation at $95^{\circ} \mathrm{C}, 20$ s of annealing at $58^{\circ} \mathrm{C}$, and $40 \mathrm{~s}$ of extension at $72^{\circ} \mathrm{C}$; and a $7 \mathrm{~min}$ final extension at $72^{\circ} \mathrm{C}$. Polymerase chain reaction products were electrophoresed through a 7\% polyacrylamide gel in $0.5 \times$ TBE buffer and the gel was stained in ethidium bromide ( $1 \mathrm{mg} / \mathrm{mL}$ ) for $20 \mathrm{~min}$. A photoimage was captured by using BiPS 3.0 (Biomedlab, Seoul, Korea).

Parental origin of abnormal 10p was confirmed by using

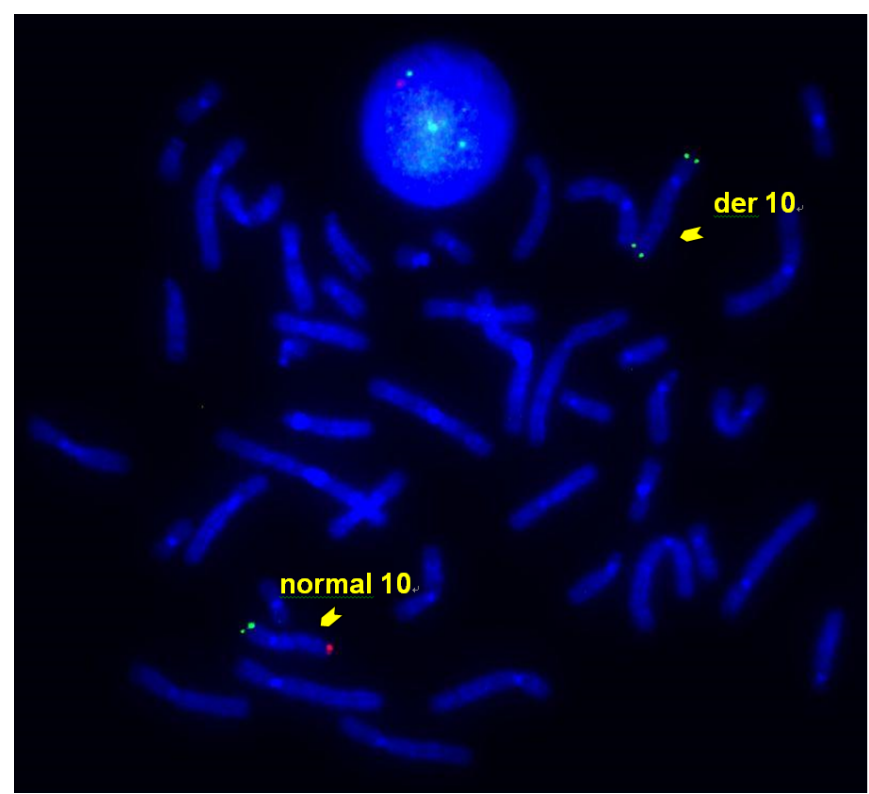

Fig. 2. Fluorescence in situ hybridization (FISH) analysis. The deletion of the terminal region of chromosome $10 \mathrm{q}$ (spectrum orange signal) and the duplicated signals for the terminal region of chromosome $10 \mathrm{p}$ on both ends of derivative (der) chromosome 10 (spectrum green signal).
A

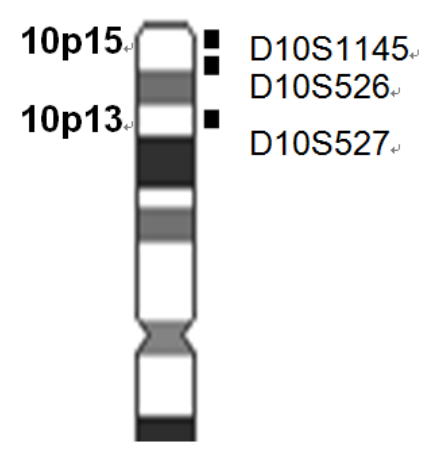

B.

D10S526 D10S1145 D10S527

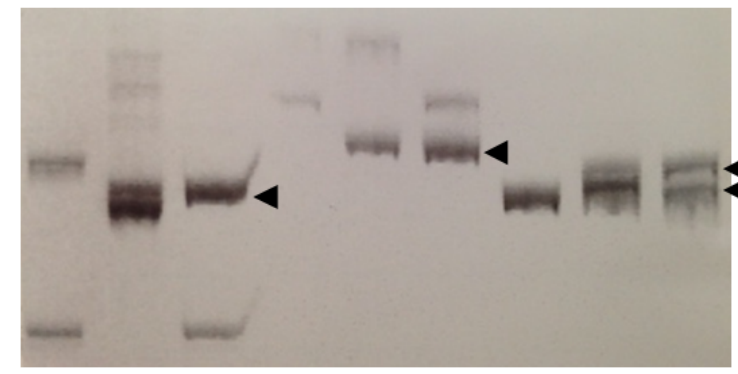

$\begin{array}{lllllllll} & \mathbf{M} & \mathbf{B} & \mathbf{F} & \mathbf{M} & \mathbf{B} & \mathbf{F} & \mathbf{M} & \mathbf{B}\end{array}$
Fig. 3. (A) An ideogram of chromosome 10 depicting three informative short tandem repeat (STR) markers of the selected markers residing on the short arm of chromosome 10. (B) Polymerase chain reaction products of STR marker analysis for the parents and newborn were electrophoresed on a $7 \%$ polyacrylamide gel. For D10S526 and D10S1145, the newborn's bands from the maternal allele are more intense (arrowhead) than bands from the paternal allele. For D10S527, the newborn's bands are from maternal alleles (arrowheads). F, father; M, mother; B, baby. 
STR markers between $10 p 15$ and 10p12. Of them, three tetranucleotide markers, D10S526 (10p15), D10S1145 (10p15) and D10S527 (10p13), were informative alleles (Fig. 3A). Each maternally derived allele for the STR markers D10S526 and D10S1145 had higher intensely than that of the paternally derived alleles. For D10S527, the newborn's bands were both from maternal alleles. This indicated that the de novo duplicated 10p segment from 10p15-p13 was from the maternal allele (Fig. 3B).

\section{Discussion}

The de novo chromosomal aberration in this case showed a typical recombinant chromosome comprising a duplication of a large segment of 10p and terminal deletion of 10q. Although soem STR markers showed informative results and the region might have additional segmental crossing-over, the duplicated segment of at least the 10p15-p13 region appears to be derived from the maternal chromosome.

A duplication-deletion chromosome is usually assumed to be the product of crossing-over between an inherited pericentric inverted chromosome and its normal homologue during meiosis I. We reviewed all 14 cases of dup(10)/del(10) cases reported previously and summarized the cytogenetic and clinical findings of those and the present case (Table 1) [1-3,9-15]. Based on STR marker analyses, the de novo duplicated chromosomal segment of this case was of maternal origin. However, we could not determine whether the duplication-deletion chromosome was generated from successive rearrangement events or a distinctive independent rearrangement in a maternal gamete. We assumed two possible mechanisms for the present case: 1) a pericentric inversion and recombinant homologous might have occurred by successive cross-over at maternal meiosis I during gametogenesis, or 2) the mother may have a premeiotic pericentric inversion by maternal germline mosaicism and duplication-deletion rearrangement might have been generated by cross-over at maternal meiosis I during gametogenesis. In cases involving other autosomes, there were two reports of de novo recombinant duplication derived from premeiotic pericentric inversion on chromosome 1 and 13, respectively $[16,17]$.

From a clinical perspective, the dup(10p)/del(10q) syndrome was characterized by hypotonia, poor feeding, moderate to severe mental retardation, microcephaly, wide sutures, frontal bossing, short nose, cleft lip and palate, camptodactyly, clinodactyly, club feet, and urogenital system [3]. According to the size of the duplicated or deleted segment, dup(10p)/del(10q) cases share similar clinical manifestation with trisomy 10p or deletion 10q syndrome (Table 2) [3]. The infant we described had a few common clinical anomalies of dup(10p) and del(10q) that are parallel to those of other reported cases with similar break points $[1,3,9]$. Deletion of $10 q 26$ is more common compared to del(10)(q25), but demonstrates a less severe phenotype. Winsor et al. [18] and Yunis and Torres de Caballero [10] suggested that the distance between the break points and the centromere determine the lengths of the duplication or deletion and affect the viability of embryos. Additionally, genital anomalies in males, such as cryptorchidism are a highly frequent phenotype associated with the deletion 10q26. Chung et al. [19] and Tanabe et al. [20] suggested that genes in the region of $10 q 25$ or $10 q 26$ are related to the regulation of the testis-determining gene, $S R Y$, or male sex differentiation.

Typical clinical manifestations are difficult to discriminate among syndromes of trisomy 10p, deletion 10q, and dup(10p)/ del(10q). Upslanting palpebral fissures in the current infant seem to be related to trisomy 10p. High arched/cleft palate and retrognathia, reported as major anomalies of trisomy 10p, were excluded in this case, while the 10p11.2-p12.2 breakpoint region is thought to be related to these anomalies [21]. Hypotonia and a suspicious polycystic kidney are related to common symptoms of dup(10p)/del(10q) syndrome. Moreover, the 10q26 region is associated with obstructive uropathy due to anomalous ureterovesical junction [22]. Various reports suggest that the $10 \mathrm{q}$ terminal deletion is related to renal and urinary tract malformation in many female cases $[3,23]$. We inferred that the deletion of the 10q26.3 region in the present case is related to the suspicious polycystic kidney in our patient. Idiopathic jaundice was observed in the case of Lansky-Shafer et al. [1]. Thrombocytopenia was also observed in the cases of Ohba et al. [2] and Roberts et al. [11]. Neonatal respiratory distress has been reported in cases of del(10q) [24]. Lozić et al. [25] examined complete trisomy 10p syndrome and described a patient's physical defects such as dolichocepahly, cleft lip and palate, microcephaly, hypotonia, flat nasal bridge, clubfoot anomaly, marbled skin, and atrial septal defect.

In conclusion, this is de novo dup(10p)/del(10q) case is rarely observed; it witll facilitate the diagnosis of similar cases of recombinant dup(10p)/del(10q) and provides clinical information for genetic counseling. 


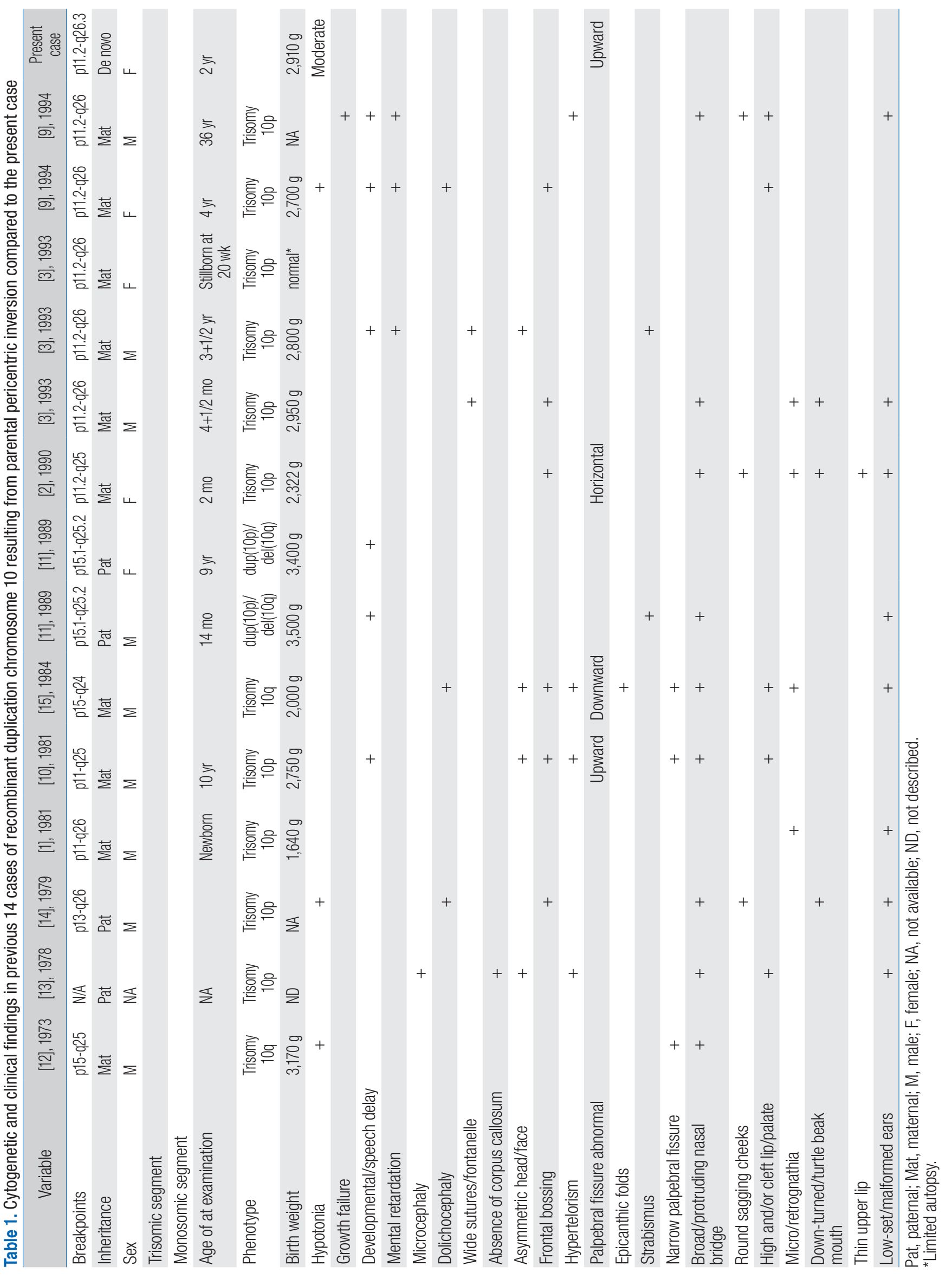




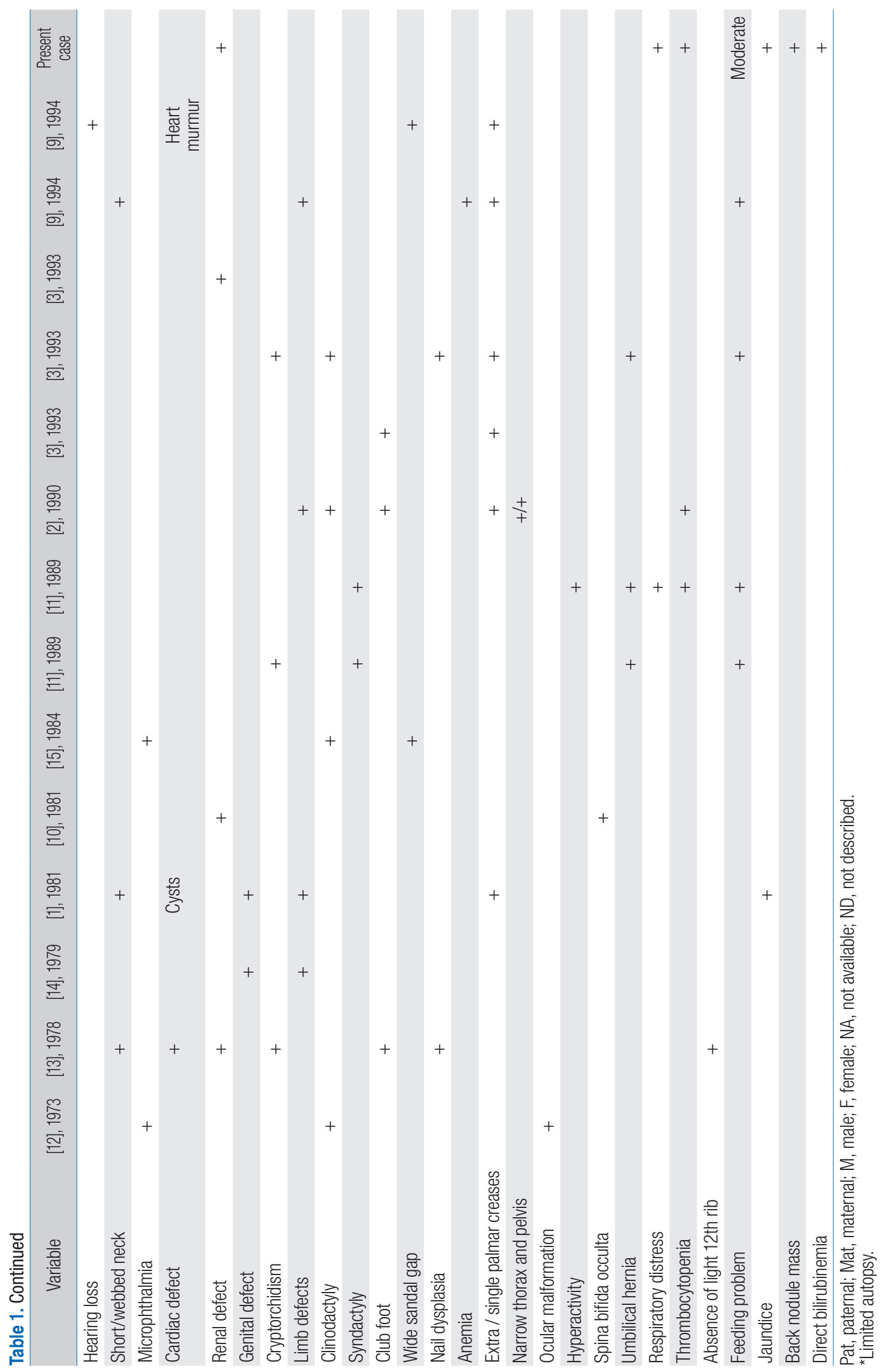


Table 2. Summary of major phenotypic anomalies of dup(10p), dup (10p)/del(10q) and del (10q) syndromes

\begin{tabular}{|c|c|c|c|}
\hline Manifestation & Dup(10p) & $\begin{array}{l}\text { Dup(10p)/ } \\
\operatorname{del}(10 q)\end{array}$ & $\operatorname{Del}(10 q)$ \\
\hline Hypotonia & + & + & + \\
\hline Microcephaly & + & & + \\
\hline Dolichocephaly & + & & \\
\hline Wide sutures & + & + & \\
\hline Frontal bossing & + & + & \\
\hline Broad/short nose & + & + & + \\
\hline \multicolumn{4}{|l|}{ Palpebral fissures } \\
\hline Up-slanting & + & & \\
\hline Horizontal & & & + \\
\hline Strabismus & & & + \\
\hline Cleft lip/palate & + & & \\
\hline Micro/retrognathia & & + & \\
\hline Down-turned mouth & + & & + \\
\hline Malformed ears & + & + & + \\
\hline Short/webbed neck & & + & + \\
\hline Cardiac defect & + & & + \\
\hline Renal defect & + & + & + \\
\hline Neonatal respiratory problem & & & + \\
\hline \multicolumn{4}{|l|}{ Genital defect } \\
\hline Cryptorchidism & & & + \\
\hline Limb defects & + & + & \\
\hline Growth failure & + & + & + \\
\hline
\end{tabular}

\section{Acknowledgements}

This study was approved by the Ethics Committee of Cheil General Hospital and Women's Health Care Center (\#CGHIRBGR-2007-02) and patient's consent was obtained.

\section{References}

1. Lansky-Shafer SC, Daniel WL, Ruiz L. Trisomy 10p produced by recombination involving maternal inversion inv(10)(pllq26). J Med Genet 1981;18:59-61.

2. Ohba K, Ohdo S, Sonoda T. Trisomy 10p syndrome owing to maternal pericentric inversion. J Med Genet 1990;27:264-6.

3. Kulharya AS, Schneider NR, Wilson GN. Three cases of dup(10p)/ del(10q) syndrome resulting from maternal pericentric inversion. Am J Med Genet 1993;47:817-9.

4. Berend $S A$, Shaffer $L G$, Bejjani BA. Pure trisomy $10 p$ involving an isochromosome 10p. Clin Genet 1999;55:367-71.

5. Granata $P$, Mazzola D, Righi R, Minelli E, Salvatoni A, Baroli $P$, et al. Molecular cytogenetics, RFLP analysis and clinical characterization of a de novo trisomy 10p case. Ann Genet 2000;43:45-50.
6. Chen Z, Meloni-Ehrig A, Palumbos JC, Guan XY, Carroll KL, Dent KM, et al. Pure trisomy 10p resulting from an extra ring chromosome: characterization by methods of advanced molecular cytogenetics. Am J Med Genet 2001;102:379-82.

7. Lewandowski RC Jr, Kukolich MK, Sears JW, Mankinen CB. Partial deletion 10q. Hum Genet 1978;42:339-43.

8. Kallioniemi A, Kallioniemi OP, Piper J, Tanner M, Stokke T, Chen L, et al. Detection and mapping of amplified DNA sequences in breast cancer by comparative genomic hybridization. Proc Natl Acad Sci U S A 1994;91:2156-60.

9. Kozma C, Meck JM. Familial 10p trisomy resulting from a maternal pericentric inversion. Am J Med Genet 1994;49:281-7.

10. Yunis $E_{1}$ Torres de Caballero 0. Duplication deficiency as the result of meiotic segregation of a maternal InV (10). Hum Genet 1981;57:71-4.

11. Roberts $P$, Williams J, Sills MA. A case of two inversion (10) recombinants in a family. J Med Genet 1989;26:461-4.

12. Dutrillaux $B$, Laurent $C_{1}$ Robert JM, Lejeune J. Pericentric inversion, inv(10), in a mother and aneusomy by recombination, inv(10), rec(10), in her son (author's transl). Cytogenet Cell Genet 1973;12:245-53.

13. Sekhon GS, Sly WS. Trisomy 10p due to familial pericentric inversion. Birth Defects 1978;XIV:422-3.

14. Nomoto $N$, Nagauchi O. A partial 10p trisomy: 46,rec(10),dup p,inv(10) (p13q26) pat. Jpn J Hum Genet 1979;24:165-8.

15. Rodriguez MT, Martin MJ, Abrisqueta JA. Familial pericentric inversion (10) and its effect on two offspring. J Med Genet 1984;21:317-9.

16. Fan YS, Jung J, Hamilton B. Small terminal deletion of $1 p$ and duplication of 1q: cytogenetics, FISH studies, and clinical observations at newborn and at age 16 years. Am J Med Genet 1999;86:118-23.

17. Habedank M. Partial trisomy $13 q 21$ toqter de novo due to a recombinant chromosome rec(13)dup q. Hum Genet 1979;52:91-9.

18. Winsor EJ, Palmer CG, Ellis PM, Hunter JL, Ferguson-Smith MA. Meiotic analysis of a pericentric inversion, inv(7) (p22q32), in the father of a child with a duplication-deletion of chromosome 7 Cytogenet Cell Genet 1978;20:169-84.

19. Chung YP, Hwa HL, Tseng LH, Shyu MK, Lee CN, Shih JC, et al. Prenatal diagnosis of monosomy 10q25 associated with single umbilical artery and sex reversal: report of a case. Prenat Diagn 1998;18:73-7.

20. Tanabe $\mathrm{S}$, Akiba T, Katoh M, Satoh T. Terminal deletion of chromosome 10q: clinical features and literature review. Pediatr Int 1999;41:565-7.

21. Mégarbané A, Gosset P, Souraty N, Lapierre JM, Korban R, Zahed L, et al. Chromosome 10p11.2-p12.2 duplication: report of a patient and review of the literature. Am J Med Genet 2001;104:204-8.

22. Leonard NJ, Harley FL, Lin CC. Terminal deletion of chromosome 10q at band 26.1: follow-up in an adolescent male with high-output renal failure from congenital obstructive uropathy. Am J Med Genet 1999;86:115-7. 
23. Scigliano S, Grégoire MJ, Schmitt M, Jonveaux PH, LeHeup B. Terminal deletion of the long arm of chromosome 10. Clin Genet 2004;65:2948.

24. Shapiro SD, Hansen KL, Pasztor LM, DiLiberti JH, Jorgenson RJ, Young $\mathrm{RS}$, et al. Deletions of the long arm of chromosome 10. Am J Med
Genet 1985;20:181-96.

25. Lozić B, Culić V, Lasan R, Tomasović M, Samija RK, Zemunik T. Complete trisomy 10p resulting from an extra stable telocentric chromosome. Am J Med Genet A 2012;158A:1778-81. 